\title{
Integration of Color and Texture Features in CBIR System
}

\author{
Hany F. Atlam \\ Computer science and \\ Engineering Dept., Faculty of \\ Electronic Engineering, \\ Menoufia University, Menouf, \\ 32952, Egypt
}

\author{
Gamal Attiya \\ Computer science and \\ Engineering Dept., Faculty of \\ Electronic Engineering, \\ Menoufia University, Menouf, \\ 32952, Egypt
}

\author{
Nawal El-Fishawy \\ Computer science and \\ Engineering Dept., Faculty of \\ Electronic Engineering, \\ Menoufia University, Menouf, \\ 32952, Egypt
}

\begin{abstract}
Nowadays, rapid and effective searching for relevant images in large image databases has become an area of wide interest in many applications. The current image retrieval system is based on text-based approaches. This system has many challenges such as it cannot retrieve images that are context sensitive and the amount of effort required to manually annotate every image, as well as the difference in human perception when describing the images, which result in inaccuracies during the retrieval process. Content-based image retrieval (CBIR) supports an effective way to retrieve images depending on automatically derived image features. It retrieves relevant images using unique image features such as texture, color or shape.
\end{abstract}

This paper presents novel methods to retrieve relevant images from large image databases. Two proposed methods are presented. The first proposed method improves the retrieval performance by identifying the most efficient gray-level cooccurrence matrix (GLCM) texture features and combine them with the appropriate Discrete Wavelet Transform (DWT) decomposition band. The second proposed method increases the system performance by combining color and texture features as one feature vector which is resulting in increasing the retrieval accuracy. The proposed methods have shown a promising and faster retrieval on a WANG image database containing 1000 color images. The retrieval performance has been evaluated with the existing systems that discussed in the literature. The proposed methods give better performance than other systems.

\section{Keywords}

CBIR, Color Histogram, GLCM, DWT, Image Retrieval, WANG image database, Euclidean distance

\section{INTRODUCTION}

With the development of the Internet and the availability of different imaging devices, the size of generated digital images is increasing rapidly [1]. When the amount of images becomes so large, it will be useless unless there is an effective tool to retrieve desired images. For this purpose, many image retrieval approaches have been developed. They are textbased and content-based [2].

In Text-based Image Retrieval (TBIR) approach, images are indexed by textual description, known as the metadata of the image, such as the date at which image was produced and a manually annotated description of the content of the image itself [3]. TBIR approach has many difficulties such as it cannot retrieve images that are context sensitive and the amount of effort required to manually annotate every image, as well as the difference in human perception when describing the images, which result in inaccuracies during the retrieval process. To overcome the shortcoming associated with TBIR system, Content-based Image Retrieval (CBIR) approach was introduced in the early 1980s [1].

In CBIR system, visual features of the image such as color, texture, shape or any other information that can be automatically extracted from the image are extracted and used to retrieve relevant images from the image database. The retrieved images are then ranked according to similarities between the query image and images in the database using a similarity matching measure [3]. A CBIR system consists of two major processes: feature extraction and similarity matching [4]. The block diagram of CBIR system is shown in Figure 1.

Feature extraction is considered the most difficult task in the retrieval process [5]. It involves extracting significant features of the image to a distinguishable extent. The similarity matching is concerned with matching extracted features to get a result that is visually similar [6]. So for a given query image, its feature vector is extracted. If the similarity distance between the feature vector of the query image and the feature vector of database images is small, then the corresponding image in the database is considered as a match for the query. The retrieval is commonly based on similarity instead of the exact match and the retrieved images are ranked depending on to a similarity index [6].

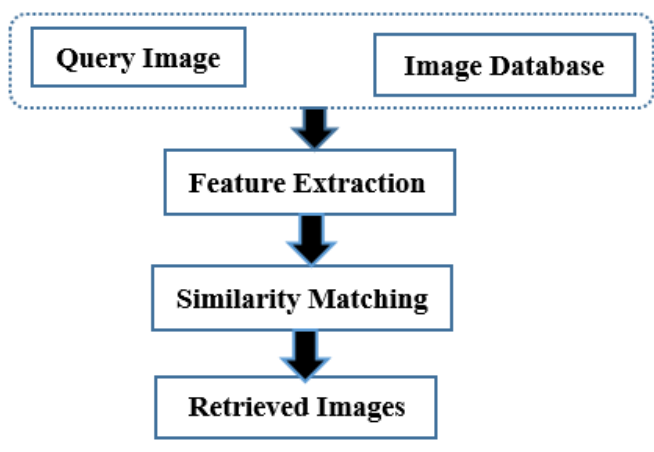

Fig 1: Block diagram of CBIR system [4]

\section{RELATED WORK}

CBIR systems describe the process of finding the images from large databases that match to a given query image using image content features. One of the problems that arise when implementing a CBIR system is how to make it a generalpurpose system. This is because the difficulty of interpreting images by users and computers, the difficulty of system evaluation, and retrieval results with different image databases [7]. 
Many CBIR systems have been successfully developed using different image features. Jhanwar et al. [8] proposed an image retrieval system that is based on motif co-occurrence matrix (MCM). This system transforms the difference between pixels into a basic graphic and estimates the probability of its occurrence in the adjacent area as the image feature. Also, Huang and Dai [9] proposed a texture-based image retrieval system which integrates the wavelet decomposition and the gradient vector. The system involves a coarse feature descriptor and a feature descriptor with each image. Both descriptors are gotten from the wavelet coefficients of the original image. The coarse feature descriptor is utilized at the first stage to immediately screen out non-promising images; the fine feature descriptor is subsequently utilized to find appropriately matched images.

Lin et al. [10] presented a Color-Texture and Color-Histogram based Image Retrieval system (CTCHIR). They proposed three image content features that based on color, texture and color distribution, as color co-occurrence matrix (CCM), the difference between pixels of scan pattern (DBPSP) and color histogram for K-mean (CHKM) respectively. They also proposed a method for the image retrieval process by integrating CCM, DBPSP, and CHKM to improve image detection rate and simplify the computation of image retrieval. Using experimental results, they found that their proposed method outperforms the Jhanwar et al.[8] and Hung and Dai [9] methods.

Rao et al. [11] presented a CTDCIRS (color-texture and dominant color based image retrieval system). They proposed integrating three features which are Motif co-occurrence matrix (MCM), the difference between pixels of scan pattern (DBPSP) and dynamic dominant color (DDC) to extract color feature. They tested their results with methods of Jhanwar et al. [8] and Hung and Dai [9] and found that their method gives superior retrieval results.

Hiremath and Pujari [12] proposed a CBIR system that is based on the color, texture and shape features by dividing the image into tiles. The features computed on tiles serve as local descriptors of color and texture features. The color and texture analysis are analyzed by using two level grid frameworks and the shape feature is used by using Gradient Vector Flow. The proposed method is compared with other systems [13] and found that their proposed retrieval system gives better performance.

Zhang [14] computed color and texture features from the image database. So, for every image, color and textures features are computed. For any query image, the color and texture features are computed. At first, the images in the database are ranked according to the color features then the top-ranked images from color features are re-ranked according to the texture features. Also, Sharma et al. [15] proposed an efficient CBIR system that used color histogram processing. The proposed methodology used color moments and color histograms. They computed color moments from the images. The color features are then used for matching between the query image and images in the database.

As a summary, working with color images and extracting its visual features have some problems. First, color images have large dimensions, also computations process are quite timeconsuming. Second, color images are sensitive to noise interference [7]. For an efficient CBIR system, a single attribute is not efficient to represent image features. Our contribution is to provide an efficient CBIR system by combining color and texture features as a single feature vector. Two new proposed methods are suggested. This result in reducing retrieval time and increasing retrieval accuracy. The proposed methods are compared with results of the methods presented by Jhanwar et al. [8], Huang and Dai [9], Rao et al. [11] and Lin et al. [10]. We found that the proposed methods give better performance.

This paper is organized as follows. The next section briefly describes feature extraction techniques that will be used in our work, the experimental evaluation is discussed in section 4 , the first proposed method is discussed in section 5, the second proposed method is discussed in Section 6, and finally the conclusion is in Section 7.

\section{FEATURE EXTRACTION TECHNIQUES}

Feature extraction is a method of extracting compact but semantically valuable information from images. This information is utilized as a signature for the image. Similar images should have identical signatures. The extracted information is used to measure the similarity between two images [16]. In this paper, There are three feature extraction techniques which are as follow:

\subsection{HSV Color Histogram}

The color feature has generally been utilized as a part of CBIR system; because of its simplicity and fast computation. The color is also an intuitive feature and plays a vital role in image matching. The extraction of color features from digital images is based on the understanding of the theory of color and the representation of color in digital images [17]. The image histogram is a graphical representation of an image. As we probably aware that the image is a set of pixels i.e. row and column, each pixel is correlated to a specific histogram bin only on the basis of its own color, so the image histogram illustrates the proportion of pixels of each color within the image [18]. Figure 2 shows how HSV color histogram is represented.

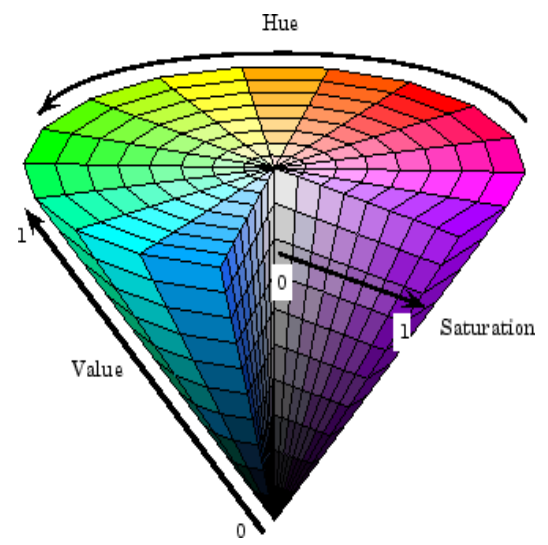

Fig 1: Representation of the HSV color descriptor [18].

HSV (Hue, Saturation, and Value) is widely used in computer graphics. The three color characteristics, hue, saturation, and value or lightness, are used to distinguish color components. This color space is the closest perception of the human eye. The generation of the HSV color space is represented According to[19], Hue defines the color by changing its angle; this angle is in the range $[0,2 \pi]$. Saturation is the depth or the purity of the color and is measured as a radial distance from the central axis with a value between 0 at the center to 1 at the outer space. Finally, Value is represented by the vertical central axis [18]. 


\subsection{Discrete Wavelet Transform (DWT)}

The texture is an essential visual feature in any CBIR system. There is no unique definition for texture; however, a scientific definition as given in [20] that is stated as "Texture is an attribute representing the spatial arrangement of the gray levels of the pixels in a region or image". Also, the texture may consist of some basic primitives, and may also show the structural arrangement of a region and the relationship of the surrounding regions Many objects in an image can only be distinguished by their texture features without any other information [21]. The commonly known texture descriptors are Wavelet Transform and co-occurrence matrices.

The wavelet transform has been widely utilized in the image processing field. Wavelet transform uses wavelet filters such as Haar Wavelet filter, Daubechie filter to transform the image [22]. Each of these filters decomposes the image into several frequencies. The Discrete Wavelet Transform (DWT) decompose the image into a lower resolution approximation image (LL), horizontal (HL), vertical (LH) and diagonal (HH) detail components [23]. This process can then be repeated to compute multi-scale wavelet decomposition, as shown in Figure 3.

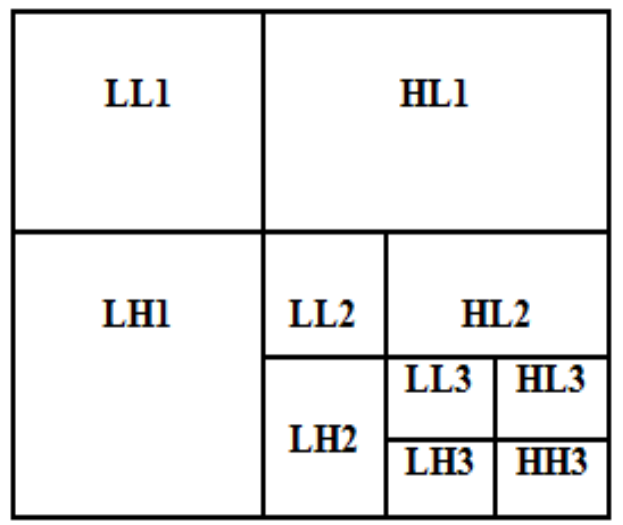

Fig 2: Representation of multi-resolution DWT [24]

\subsection{Gray Level Co-occurrence Matrix (GLCM)}

The gray level co-occurrence matrix (GLCM) determined by Haralick can describe certain features about the spatial distribution of the gray levels in the texture image [25]. GLCM indicates how often a pixel with the intensity value $\mathrm{i}$ occur in a specific spatial relationship to a pixel with the value $j$. In GLCM, each element $p(i, j)$ is the sum of the number of times that the pixel with value $i$ occurred in the specified spatial relationship to a pixel with the value $\mathrm{j}$. By default, the spatial relationship is known as the pixel of interest and the pixel to its horizontally adjacent.

Haralick [25] proposed 14 texture features and its derivatives, which almost give about 22 texture features. These features include: autocorrelation, contrast, maximal correlation coefficient, correlation, cluster prominence, cluster shade, dissimilarity, energy, entropy, homogeneity, maximum probability, sum of squares, sum average, sum variance, sum entropy, difference variance, difference entropy, information measure of correlation1, information measure of correlation2, inverse difference, inverse difference normalized and inverse difference moment normalized.

\section{EXPERIMENTAL EVALUATION}

To evaluate the performance of proposed methods, there are many things to carry out as discussed in the following sections.

\subsection{Simulation Environment}

Feature extraction algorithms and similarity measurement methods are coded using MATLAB on Intel(R) Core(TM) i5 CPU with 4GB RAM running Windows 7 and several experiments are carried out to evaluate the performance of the system. In our experiments, The WANG image database is being used to evaluate the performance of the system. The WANG database is a general-purpose image database that includes a subset of the Corel database of 1000 images. The images are of the size $256 \times 384$ images and are subdivided into 10 categories of 100 images each [27]. These categories include African People, Beach, Building, Buses, Dinosaurs, Elephants, Flowers, Horses, Mountains and Food. Some examples of the WANG image database are shown in Figure 4. The WANG database was used extensively to test the different features because the size of the database and the availability of class information allows for performance evaluation [10].

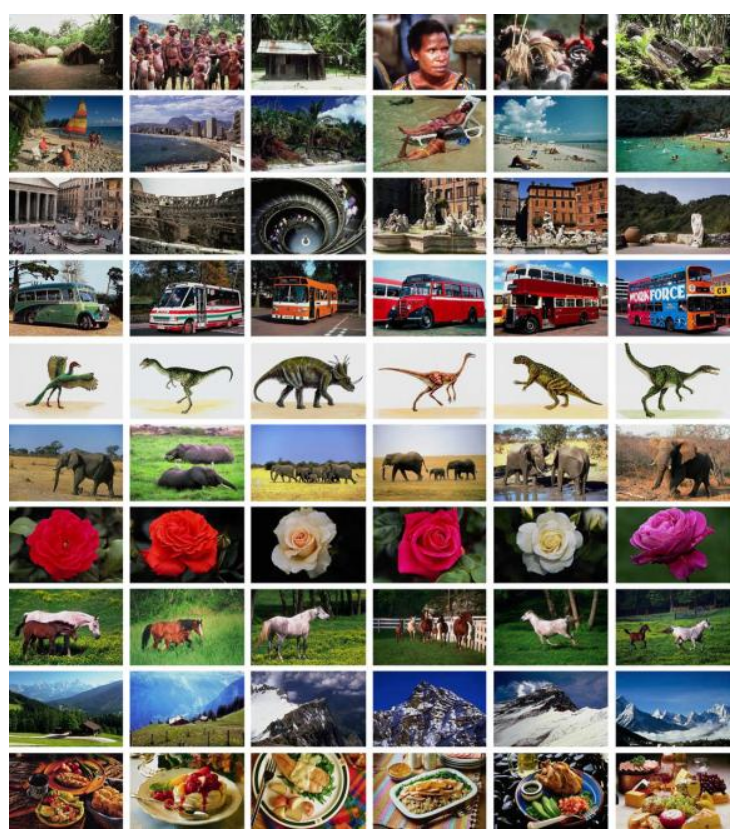

Fig 3: Some examples of WANG image database [10]

\subsection{Similarity Measurement}

To retrieve relevant images from the database, a similarity measurement is required to compute similarities between a pair of images. This distance matrix is generally used for similarity matching in image retrieval system because of its efficiency and effectiveness. The Euclidean distance is the most common distance matrix. It simply computes the root of square differences between coordinates of a pair of images $h$ and $\mathrm{g}$. The general formula for this measure is [26].

$$
\mathrm{D}=\sqrt{\sum(\mathrm{h}-\mathrm{g})^{2}}
$$

\subsection{Performance Evaluation}

In order to test retrieval effectiveness of the proposed methods, a test is carried out by selecting some random images as query images and compute the corresponding retrieve results. In this paper, the performance metric that will be used to evaluate the system is the retrieval accuracy. 
The retrieval accuracy is defined as the ratio of the number of relevant images retrieved to the total number of images retrieved expressed in percentage [28].

Accuracy $=\frac{\text { No of relevant images retrieved }}{\text { Total number of images retrieved }}$

Where the total number of images retrieved $=$ number of relevant images + number of irrelevant images. For example, if the query results in 20 images with 15 relevant images to the query image, then the accuracy of the retrieval process is $(15 / 20)=0.75[28]$.

The experiments are performed to evaluate the image retrieval system. Five images are selected randomly from each category from the WANG image database and used it as query images. For each retrieval, the top-most 20 images are retrieved to estimate the retrieval accuracy. The average retrieval accuracy of the five tries is computed for each category of the WANG database.

\section{FIRST PROPOSED METHOD}

Texture, like color, is a powerful low-level feature for image search and retrieval applications [29]. Our proposed method is based on texture features to retrieve relevant images from the image database. This method is divided into two stages: The first stage is concerned with selecting the most efficient GLCM texture features for the retrieval process. As said earlier, Haralick proposed 14 GLCM texture features and its derivatives, which almost give 22 texture features [25]. Extracting 22 different texture features for each database image in and for each query image takes large time. So that our goal is to identify the most efficient GLCM texture features that can be used in CBIR system to increase the retrieval performance. This can be done by evaluating the retrieval accuracy of each GLCM texture feature individually. Then, the most efficient GLCM texture features can be determined. The proposed methodology is shown in Figure 5.

The experimental results showed that the most efficient GLCM texture features that can be used in the image retrieval system are: autocorrelation, maximal correlation coefficient, dissimilarity, sum average and information measure of correlation2. These six GLCM texture features give high retrieval accuracy that is nearby the retrieval accuracy given by all 22 GLCM texture features together. As shown in Table 1 , the average retrieval accuracy using 22 GLCM texture features was 0.35. However, after defining the most efficient texture features from GLCM, the average retrieval accuracy becomes 0.33 . So, the retrieval accuracy still high, but the processing time that is required for the feature extraction process is absolutely decreased.

The second stage of the proposed method was to combine efficient GLCM texture features that are identified in the first stage of the DWT decomposition to increase retrieval accuracy and also decrease processing time required for the feature extraction process. This is because DWT decomposition works only with one-quarter of the image.
Step1. Read the query image.

Step2. Convert the query image from RGB into gray scale.

Step3. Compute GLCM texture feature of the query image.

Step4. Compute GLCM texture feature for all images in the database.

Step5. Compute the Euclidean distance by comparing the query image GLCM texture features to that of each image in the database.

Step6. Sort images in the database in order of descending Euclidean distance values and retrieve the top-most 20 images.

\section{Fig 4: Proposed methodology to identify the most efficient} GLCM texture features

The experimental results showed that the horizontal HL band is the more appropriate DWT band that can work efficiently with the image retrieval system. It also showed that combining efficient GLCM texture features with DWT increases the retrieval accuracy. As shown in Table 1, the average retrieval accuracy using only efficient GLCM texture features was 0.33 , but with DWT decomposition, the average retrieval accuracy increased to 0.43 .

Table 1. Retrieval accuracy of the first proposed method

\begin{tabular}{|c|c|c|c|}
\hline Category & $\begin{array}{c}\text { All } \\
\text { GLCM } \\
\text { features }\end{array}$ & $\begin{array}{c}\text { Efficient } \\
\text { GLCM } \\
\text { features }\end{array}$ & $\begin{array}{c}\text { Efficient } \\
\text { GLCM } \\
\text { With DWT }\end{array}$ \\
\hline African & 0.15 & 0.20 & 0.45 \\
\hline Beach & 0.25 & 0.30 & 0.45 \\
\hline Building & 0.20 & 0.10 & 0.30 \\
\hline Buses & 0.20 & 0.25 & 0.50 \\
\hline Dinosaurs & 0.98 & 0.98 & 0.98 \\
\hline Elephants & 0.70 & 0.35 & 0.30 \\
\hline Flowers & 0.35 & 0.40 & 0.50 \\
\hline Horses & 0.30 & 0.30 & 0.35 \\
\hline Mountains & 0.20 & 0.20 & 0.30 \\
\hline Food & 0.15 & 0.20 & 0.20 \\
\hline Average & 0.35 & 0.33 & 0.43 \\
\hline & & &
\end{tabular}

Fig 5 shows the result of retrieved images using efficient GLCM texture features. The query image belongs to flowers category from the WANG image database. The number of relevant images to the query image are 10 


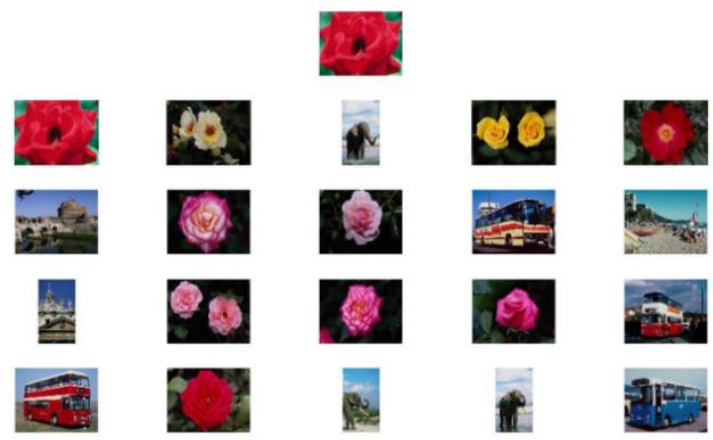

Fig 5: Retrieved images using efficient GLCM

Figure 7 shows the result of retrieved images using efficient GLCM texture features with DWT decomposition. The query image belongs to dinosaurs category from the WANG image database. All retrieved images are relevant to the query image.
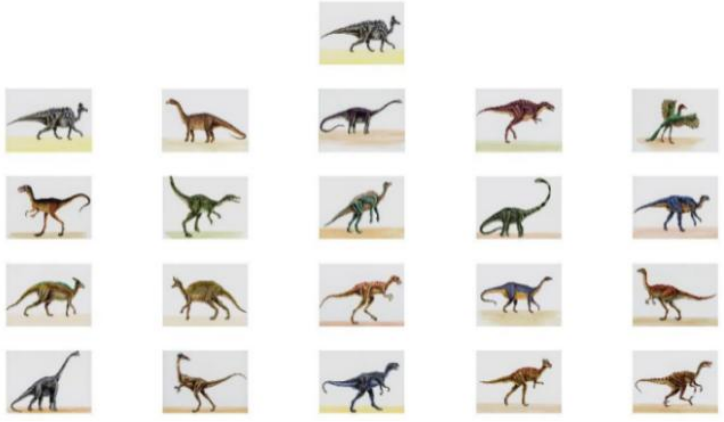

Fig 6: Retrieved images using efficient GLCM with DWT

\section{SECOND PROPOSED METHOD}

Because of its simplicity and fast computation, the color feature has generally been utilized as part of any CBIR system. The color is an intuitive feature that plays a vital role in images matching [10]. Texture also is a powerful low-level feature for image search and retrieval applications [29]. Our proposed method is based on combining color and texture features to increase the image retrieval accuracy. Based on the comparative study made by [4], HSV color histogram is the most efficient color feature extraction technique for the image retrieval system. The results obtained from the first proposed method, which defines efficient GLCM texture features with DWT HL band, are utilized and combined with the HSV color histogram to constitute a single feature vector. The proposed methodology is shown in Figure 8.

The experimental results showed that combining color with texture features improved the average retrieval accuracy of the image retrieval system. As shown in Table 2, using the HSV color histogram and efficient GLCM texture features with the DWT HL band gives an average retrieval accuracy of 0.74 and 0.43 respectively. While with combing them together, the average retrieval accuracy increased to 0.77
Step1. Read the query image.

Step2. Convert the query image from RGB into HSV.

Step3. Compute HSV Color Histogram for the query image.

Step4. Compute GLCM texture feature on DWT HL band of the query image.

Step5. Form the Feature vector of the query image.

Step6. Compute HSV Color Histogram of images in the database.

Step 7. Compute GLCM texture feature on DWT HL band of images in the database.

Step8. Form the Feature vector of images of the database.

Step9. Compute a Euclidean distance by comparing the feature vector of the query image with the feature vector of images in the database.

Step10. Sort images in the database in order of descending Euclidean distance values and retrieve the top-most 20 images.

Fig 7: Proposed methodology to combine HSV color histogram with the most efficient GLCM texture features and DWT HL band.

Table 2. Retrieval accuracy of the second proposed method

\begin{tabular}{|c|c|c|c|}
\hline Category & $\begin{array}{c}\text { HSV color } \\
\text { Histogram } \\
{[4]}\end{array}$ & $\begin{array}{c}\text { Efficient } \\
\text { GLCM } \\
\text { With DWT }\end{array}$ & $\begin{array}{c}\text { Proposed } \\
\text { Method }\end{array}$ \\
\hline African & 0.96 & 0.45 & 0.97 \\
\hline Beach & 0.60 & 0.45 & 0.67 \\
\hline Building & 0.57 & 0.30 & 0.67 \\
\hline Buses & 0.83 & 0.50 & 0.80 \\
\hline Dinosaurs & 0.98 & 0.98 & 0.99 \\
\hline Elephants & 0.47 & 0.30 & 0.50 \\
\hline Flowers & 0.98 & 0.50 & 0.95 \\
\hline Horses & 0.94 & 0.35 & 0.97 \\
\hline Mountains & 0.25 & 0.30 & 0.37 \\
\hline Food & 0.85 & 0.20 & 0.82 \\
\hline Average & 0.74 & 0.43 & 0.77 \\
\hline
\end{tabular}

To demonstrate the superiority of the proposed methods, a comparison between proposed methods with other methods in existing systems are carried out in term of retrieval accuracy. The proposed work was compared with the work of Jhanwar et al. [8], Hung and Dai [9], CTDCIRS [11] and SCBIRS [10] as shown in Table 3. It is obvious that the proposed methods give better average retrieval accuracy than other systems. 
Table 3. Retrieval accuracy of the proposed method with other systems

\begin{tabular}{|c|c|c|c|c|c|}
\hline Category & $\begin{array}{c}\text { Jhanw } \\
\text { ar et al. } \\
{[\mathbf{8}]}\end{array}$ & $\begin{array}{c}\text { Huang } \\
\text { \&Dai's } \\
{[\mathbf{9}]}\end{array}$ & $\begin{array}{c}\text { CTDCI } \\
\text { RS [11] }\end{array}$ & $\begin{array}{c}\text { SCBI } \\
\text { RS } \\
{[\mathbf{1 0}]}\end{array}$ & $\begin{array}{c}\text { Proposed } \\
\text { Method }\end{array}$ \\
\hline African & 0.45 & 0.43 & 0.56 & 0.68 & 0.97 \\
\hline Beach & 0.40 & 0.45 & 0.54 & 0.54 & 0.67 \\
\hline Building & 0.37 & 0.41 & 0.61 & 0.56 & 0.67 \\
\hline Buses & 0.74 & 0.85 & 0.89 & 0.89 & 0.80 \\
\hline Dinosaurs & 0.92 & 0.59 & 0.98 & 0.99 & 0.99 \\
\hline Elephants & 0.30 & 0.43 & 0.58 & 0.66 & 0.50 \\
\hline Flowers & 0.85 & 0.90 & 0.90 & 0.89 & 0.95 \\
\hline Horses & 0.57 & 0.59 & 0.78 & 0.80 & 0.97 \\
\hline Mountain & 0.30 & 0.27 & 0.51 & 0.52 & 0.37 \\
\hline Food & 0.37 & 0.43 & 0.69 & 0.73 & 0.82 \\
\hline Average & 0.53 & 0.55 & 0.70 & 0.73 & 0.77 \\
\hline
\end{tabular}

Figure 8 shows the result of retrieved images using HSV color histogram. The query image belongs to food category from the WANG image database. The number of relevant images for this query is 17 .

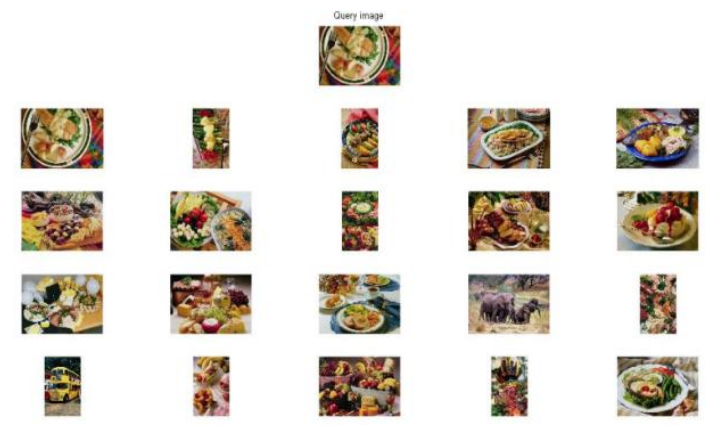

Fig 8: Retrieved images using HSV color histogram

Fig 9 shows the result of retrieved images using HSV color histogram with efficient GLCM texture features and DWT. The query image belongs to buses category from the WANG image database. All retrieved images are relevant to the query image.

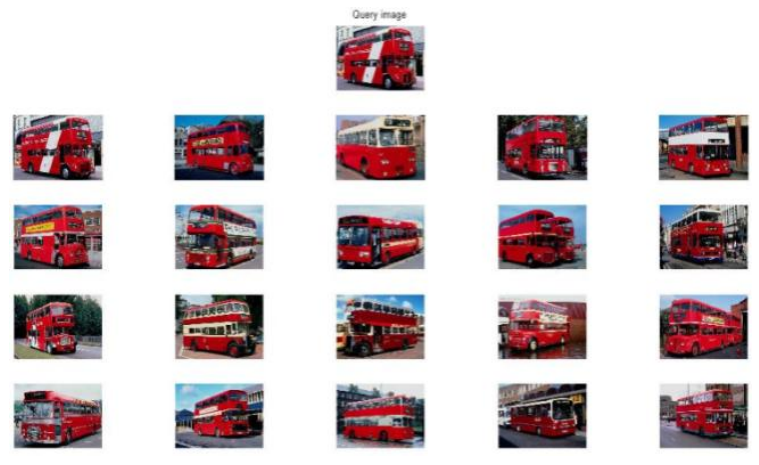

Fig 9: Retrieved images using HSV color histogram with efficient GLCM texture features and DWT

\section{CONCLUSION}

In this paper, two novel methods are presented to enhance the retrieval performance of the image retrieval system. The first proposed method defined the most efficient GLCM texture features for the retrieval process. It also increased the retrieval accuracy by combining the most efficient GLCM texture features with DWT decomposition. The second proposed method combined color and texture features to improve the retrieval performance. This method combined the HSV color histogram with the most efficient GLCM texture features and DWT. Experimental results of the proposed methods, that are performed on the WANG image database, indicated that the average retrieval accuracy increased to 0.77 . It also indicated that the proposed methods outperform Huang and Dai's Jhanwar's, Rao's and Lin's methods. In the future, we plan to evaluate proposed methods with other large general-purpose image databases. In addition, we plan to use other feature extraction techniques and apply it in the cloud-computing environment containing millions of images.

\section{REFERENCES}

[1] S. Pattanaik and D. Bhalke, "Beginners to Content-Based Image Retrieval," Ijsret.Org, vol. 1, no. May, pp. 40-44, 2012.

[2] Y. Chen, J. Z. Wang, and R. Krovetz, "CLUE: Clusterbased retrieval of images by unsupervised learning," IEEE Trans. Image Process., vol. 14, no. 8, pp. $1187-$ 1201,2005

[3] J. Z. Wang, J. Li, and G. Wiederholdy, "SIMPLIcity: Semantics-sensitive integrated matching for picture libraries," Lect. Notes Comput. Sci. (including Subser. Lect. Notes Artif. Intell. Lect. Notes Bioinformatics), vol. 1929, pp. 360-371, 2000.

[4] Hany F. Atlam, G. Attiya, and N. El-Fishawy, "Comparative Study on CBIR based on Color Feature," Int. J. Comput. Appl., vol. 78, no. 16, pp. 975-8887, 2013.

[5] E. L. Hall et al., "A Survey of Preprocessing and Feature Extraction Techniques for Radiographic Images," IEEE Trans. Comput., vol. C-20, no. 9, pp. 1032-1044, 1971.

[6] F. Z. Rangari and U. Ramarao, "Searching by Content based image retrieval through combined features," vol. 1, no. 2, pp. 85-93, 2013.

[7] J. Afifi Ahmed and M. Ashour Wesam, "Image Retrieval Based on Content using Color Feature," ISRN Comput. Graph., vol. 341-342, pp. 560-564, 2012.

[8] N. Jhanwar, S. Chaudhuri, G. Seetharaman, and B. Zavidovique, "Content based image retrieval using motif cooccurrence matrix," Image Vis. Comput., vol. 22, no. 14, pp. 1211-1220, 2004.

[9] P. W. Huang and S. K. Dai, "Image retrieval by texture similarity," Pattern Recognit., vol. 36, no. 3, pp. 665679, 2003.

[10] C.-H. Lin, R.-T. Chen, and Y.-K. Chan, "A smart content-based image retrieval system based on color and texture feature," Image Vis. Comput., vol. 27, no. 6, pp. 658-665, 2009 .

[11] M. B. Rao, B. P. Rao, and A. Govardhan, "CTDCIRS: Content based Image Retrieval System based on Dominant Color and Texture Features," Int. J. Comput. Appl., vol. 18, no. 6, pp. 40-46, 2011.

[12] P. S. Hiremath and J. Pujari, "Content Based Image Retrieval based on Color, Texture and Shape features using Image and its complement," Int. J. Comput. Sci. Secur., vol. 1, no. 4, pp. 25-35, 2007.

[13] J. Li, J. Wang, and G. Wiederhold, "IRM: integrated region matching for image retrieval," Proc. ACM Int Conf. Multimed., pp. 147-156, 2000. 
[14] D. Zhang, "Improving Image Retrieval Performance by Using Both Color and Texture Features," Third Int. Conf. Image Graph. pp. 172-175, 2004.

[15] S. Neetu Sharma, S. Paresh Rawat, and S. Jaikaran Singh, "Efficient CBIR Using Color Histogram Processing," Signal Image Process. An Int. J., vol. 2, no. 1, pp. 94-112, 2011.

[16] V. Kushwah and A. Agrawal, "Study on Query Based Clustering Technique for Content Based Image Retrieval," IJREAT Int. J. Res. Eng. Adv. Technol., vol. 2, no. 1, pp. 1-6, 2014.

[17] Singha, M., \& K.Hemachandran, "Content Based Image Retrieval using Color and Texture," Signal Image Process. An Int. J., vol. 3, no. 1, pp. 39-57, 2012.

[18] Y. An, M. Riaz, and J. Park, "CBIR based on adaptive segmentation of HSV color space," 12th Int. Conf. Comput. Model. Simul., pp. 248-251, 2010.

[19] R. Chakravarti and X. M. X. Meng, "A Study of Color Histogram Based Image Retrieval," 2009 Sixth Int. Conf. Inf. Technol. New Gener., pp. 1323-1328, 2009.

[20] [20] Institute of Electrical and Electronics Engineers (IEEE), "IEEE Standards Glossary of Image Processing and Pattern Recognition Terminology," no. 610.4-1990, 1990.

[21] C. H. Wei, Y. Li, W. Y. Chau, and C. T. Li, "Trademark image retrieval using synthetic features for describing global shape and interior structure," Pattern Recognit., vol. 42, no. 3, pp. 386-394, 2009.

[22] J. R. Smith and S.-F. Chang, "VisualSEEk: A Fully Automated Content-based Image Query System," Proc. fourth ACM Int. Conf. Multimed. - Multimed. '96, pp. 87-98, 1996.

[23] J. R. Smith and S.-F. C. S.-F. Chang, "Transform features for texture classification and discrimination in large image databases," Proc. 1st Int. Conf. Image Process., vol. 3, pp. 407-411, 1994.

[24] D. A. Kumar and J. Esther, "Comparative Study on CBIR based by Color Histogram, Gabor and Wavelet Transform," Int. J. Comput. Appl., vol. 17, no. 3, pp. 3744, 2011.

[25] R. Haralick, K. Shanmugan, and I. Dinstein, "Textural features for image classification," IEEE Transactions on Systems, Man and Cybernetics, vol. 3. pp. 610-621, 1973.

[26] L. Wang, Y. Zhang, and J. Feng, "On the Euclidean Distances of Images," IEEE Trans. Pattern Anal. Mach. Intell., vol. 27, no. 8, pp. 1334-1339, 2005.

[27] J. Li and J. Z. Wang, "Automatic linguistic indexing of pictures by a statistical modeling approach," IEEE Trans. Pattern Anal. Mach. Intell., vol. 25, no. 9, pp. 10751088, 2003

[28] A. Chadha, S. Mallik, and R. Johar, "Comparative Study and Optimization of Feature-Extraction Techniques for Content based Image Retrieval," Int. J. Comput. Appl., vol. 52, no. 20, pp. 35-42, 2012.

[29] B. S. Manjunath, J. R. Ohm, V. V. Vasudevan, and A. Yamada, "Color and texture descriptors," IEEE Trans. Circuits Syst. Video Technol., vol. 11, no. 6, pp. 703 $715,2001$. 